\title{
PROOF OF AN INEQUALITY FOR TRIGONOMETRIC POLYNOMIALS
}

\author{
G. K. KRISTIANSEN
}

\begin{abstract}
Let a trigonometric polynomial have only real roots. An inequality is proved, relating the average value of the trigonometric polynomial between two consecutive roots to its maximal absolute value.
\end{abstract}

Consider the functional

$$
f(T)=\max _{a . b} \int_{a}^{b} d x|T(x)| /(b-a) \max _{|x| \leqq \pi}|T(x)|,
$$

where $T$ is a trigonometric polynomial (in the following abbreviated tp) of degree $n \geqq 1$ with real coefficients and $2 n$ real roots, and $a$ and $b$ are two of these $(a \neq b)$.

Evidently, $f(T) \leqq 1$. I shall prove that $f(T) \leqq 2 / \pi$. As a corollary we have Erdös' conjecture [1] that

$$
\int_{-\pi}^{\pi} d x|T(x)| \leqq 4 \cdot \max _{|x| \leqq \pi}|T(x)| .
$$

A maximizing tp $T$ for $f$ is easily shown to exist. We can assume $\max _{|x| \leqq \pi}|T(x)|=1$. Let $a$ and $b$ be the maximizing roots (we can assume $T(x) \neq 0$ for $a<x<b)$.

$T$ may have multiple roots. Assume first that $a=b$. Consider a fundamental sequence of tps $\left\{T_{j}\right\}$ defining $T$, so that each $T_{j}$ has maximal absolute value 1 . Then $\max _{|x| \leqq n}\left|T_{j}^{\prime}(x)\right| \leqq n$ (Bernshtein), and $\int_{a_{j}}^{b_{j}} d x\left|T_{j}(x)\right| \leqq$ $n \cdot\left(\left(b_{j}-a_{j}\right) / 2\right)^{2}$, so that $f\left(T_{j}\right) \rightarrow 0$ for $j \rightarrow \infty$, a contradiction. A multiple root for $T$ with multiplicity $m$ will have multiplicity $m-1$ as root for $T^{\prime}$. Between two consecutive multiple roots for $T, T^{\prime}$ will have at least one root, but $T^{\prime}$ cannot have more than 1 root here since, otherwise, $T^{\prime}$ would have more than $2 n$ roots. Therefore $T^{\prime}$, like $T$, has exactly $2 n$ real roots.

To prove $f(T)=2 / \pi$ we need only prove that a real constant $c$ exists, for which $T(x)=\sin n(x-c)$.

Received by the editors November 21, 1972.

AMS (MOS) subject classifications (1970). Primary 26A82.

(c) American Mathematical Society 1974 
The general method of proof consists in showing that if $T$ has another form, it is possible to choose a tp $t$ of degree at most $n$, so that $f(T+\varepsilon t)>f(T) ; \varepsilon$ is a small positive real number. $t$ must, of course, be chosen so that $T+\varepsilon t$ still has $2 n$ real roots.

We consider changes of the first order in $\varepsilon$. The change $\varepsilon \delta_{n}$ of the numerator of $f$ is determined from

$$
\delta_{n}=\int_{a}^{b} d x t(x) .
$$

The change $\varepsilon \delta_{d}$ of the denominator of $f$ is determined from

$$
\delta_{d}=(b-a) \cdot \max _{j}\left\{t\left(z_{j}\right) \operatorname{sign}\left(T\left(z_{j}\right)\right)\right\}+\delta_{b}-\delta_{a},
$$

where the sign of $T$ is chosen so that $T(x)>0$ for $a<x<b$, and $z_{j}$ are the points in which $|T(x)|$ assumes its maximal value 1 . Moreover, $\delta_{b}=0$ for $t(b)=0$, and $\delta_{b}=-t(b) / T^{\prime}(b)$ for $t(b) \neq 0$ (we shall have $t(b) \neq 0$ only if $\left.T^{\prime}(b) \neq 0\right)$; similarly for $a$.

The inequality $f(T+\varepsilon t)>f(T)$ is, for sufficiently small $\varepsilon$, equivalent to

$$
\delta_{n}>\delta_{d} \cdot f(T) \text {. }
$$

In the following we mostly specify $t$ by indicating its root set or, rather, describing how this set differs from the root set of $T$. Note that $t$, apart from a multiplicative constant, is uniquely determined from its root set $\left\{y_{j}\right\}$ by $t(x)=\prod_{j=1}^{2 n} \sin \left(\left(x-y_{j}\right) / 2\right)$. For simplicity we shall sometimes refer to a nonroot as a root of multiplicity zero. A root interval in which $|T(x)|$ does not assume its maximal value will be termed a regular interval.

(A) Consider first the case where $(a, b)$ is regular.

(A1) $T(x)$ has a root $y$ of multiplicity $m_{y}>1$. If $y$ equals $a$ or $b, y=a$ (say), we let $t$ have the same roots as $T$, but $a$ with multiplicity $m_{a}-1$ and $b$ with multiplicity $m_{b}+1$. We require $t(x)>0$ for $a<x<b$. Then, according to (1), $\delta_{n}>0$; according to (2), $\delta_{d}<0$.

If $y \neq a, b$, we let $t$ have the same roots as $T$, except for $a, b$, and $y$, which now shall be roots of multiplicity $m_{a}+1, m_{b}+1$, and $m_{y}-2$, respectively. As before, we require $t(x)>0$ for $a<x<b$. Then $\delta_{n}>0$ and $\delta_{d}<0$.

(A2) $T(x)$ has no multiple roots. Assume that $T$ has another regular interval $\left(x_{1}, x_{2}\right)$. If $x_{2}=a, t$ and $T$ should have the same roots except for $x_{1}$, which is not a root of $t$, and $b$, a double root of $t$. Similarly for $x_{1}=b$. Otherwise, $t$ and $T$ should have the same roots except for $x_{1}$ and $x_{2}$, which are nonroots of $t$, and $a$ and $b$, which are double roots of $t$.

We are left with the case in which $(a, b)$ is the only regular interval. Consider the tp $U(x)=T\left(\frac{1}{2}(a+b)+x\right)-T\left(\frac{1}{2}(a+b)-x\right)$. We have $U(x)=$ $-U(-x)$ for all $x$, in particular $U(0)=U(\pi)=0$. Besides $U( \pm(b-a) / 2)=0$. 
In the points where $\left|T\left(\frac{1}{2}(a+b)+x\right)\right|=1, U(x) \cdot T\left(\frac{1}{2}(a+b)+x\right) \geqq 0$. We can assume that $U$ has a zero between $(b-a) / 2$ and the first such point. Let now $x$ increase starting from $x=(b-a) / 2$ and, pairing each of the points described above with the monotony interval to its left, we see that $U$ must have at least $2 n+1$ roots and therefore vanish identically. It is easily seen that the possibility of $U$ having double roots does not invalidate this result.

We may set $(a+b) / 2=0$ and write $T(x)=\cos n v$ with $\cos v=\cos ^{2} u$. $\cos x-\sin ^{2} u$ and $0<u<\pi / 4 n$. We have

$$
f(T)=\frac{1}{b} \int_{0}^{b} d x \cdot \cos n v .
$$

The connection between $b$ and $u$ is

$$
b=\arccos \frac{\sin ^{2} u+\cos (\pi / 2 n)}{\cos ^{2} u}=2 \cdot \arccos \left(\frac{\cos (\pi / 4 n)}{\cos u}\right) .
$$

After some manipulation,

$$
f(T)=\frac{2 n}{\arccos (\cos (\pi / 4 n) / \cos u)} \int_{u}^{\pi / 4 n} d v \sin 2 n v \cdot \arccos \left(\frac{\cos v}{\cos u}\right) .
$$

Now, the ratio $\arccos (\cos v / \cos u) / \arccos (\cos (\pi / 4 n) / \cos u)$ is easily shown to increase with decreasing $u$ for fixed $v$. Then $f(T)$ increases with decreasing $u$, a contradiction.

(B) We now know that $|T(x)|$ assumes its maximal value in $(a, b)$.

(B1) Assume first that $T$ has two different multiple roots, $x_{1}$ and $x_{2}$. The roots of $t$ shall be the same as those of $T$, except for $a$, whose multiplicity is increased by 1 , if $a$ is a simple root, and decreased by 1 , if $a$ equals $x_{1}$ or $x_{2}$, and similarly for $b$; furthermore the root $z \in(a, b)$ of $T^{\prime}$ shall be a double root for $t$, and if $x_{1}$ or $x_{2}$ differs from $a$ and $b$, its multiplicity as root for $t$ is decreased by $2 ; t(x) \geqq 0$ for $a \leqq x \leqq b$.

Similarly, if $T$ has a multiple root $x_{1}$ and a regular interval $\left(x_{2}, x_{3}\right)$ for which $x_{1}$ is not an endpoint, we choose the roots of $t$ as above, except for $x_{2}$ and $x_{3}$, whose multiplicities are reduced by 1 . If $b=x_{2}$ (resp. $a=x_{3}$ ) the multiplicity of $b$ (resp. $a$ ) is unchanged, otherwise it is increased by 1 .

(B2) Let $T$ have exactly one multiple root, which we can put equal to 0 . If $a=0$ we put $t(x)=T^{\prime}(x)$. If $b=0$ we put $t(x)=-T^{\prime}(x)$. In both cases $\delta_{n}=0$ and $\delta_{d}=-1$, so that (3) is satisfied. Thus, neither $a$ nor $b$ is a multiple root of $T$.

We know that if a root interval $\left(x_{1}, x_{2}\right)$ exists, in which $|T(x)|$ does not assume its maximal value, we must have either $x_{1}=0$ or $x_{2}=0$. Replacing 
$T(x)$ by $T(-x)$, if necessary, we can assume that $x_{1}=0$. The least positive root of $T^{\prime}$ is denoted by $r$.

Putting $t(x)=-T^{\prime}(x) \cdot \cot (x / 2)$ we get

$$
\delta_{n}=-\int_{a}^{b} d x \frac{T(x)}{1-\cos x} ; \quad \delta_{d}=\cot (b / 2)-\cot (a / 2) .
$$

The inequality (3) becomes

$$
\int_{a}^{b} d x T(x) g(x)<0,
$$

where $g(x)=h(x)-(b-a)^{-1} \int_{a}^{b} d y h(y)$ and $h(x)=(1-\cos x)^{-1}$. As shown below, it suffices to prove

$$
\left(T\left(x_{1}\right)=T\left(x_{2}\right) \wedge\left|x_{1}\right|<\left|x_{2}\right|\right) \Rightarrow\left|T^{\prime}\left(x_{1}\right)\right| \leqq\left|T^{\prime}\left(x_{2}\right)\right|,
$$

where $x_{1}$ and $x_{2}$ belong to the same root interval $[a, b]$ for $T$, and $|x|$ denotes the distance from $x$ to the set $\{2 p \pi ; p$ integral $\}$. Since in (5) only absolute values of abscissas are involved, and since $g(x)$ is an even function, we can restrict ourselves to the case $0<(a+b) / 2 \leqq \pi$, implying $|a| \leqq|b|$. As before, we assume $T(x)>0$ for $a<x<b$.

Assume that (5) is true. $T^{\prime}$ has exactly one root (call it $z$ ) in $(a, b)$. Assume first that $\pi<b$. If then $z>\pi$ also, we obtain by integration from $(x, T)=(z, 1)$ that

$$
\left(T\left(x_{1}\right)=T\left(x_{2}\right) \wedge x_{1}<x_{2}\right) \Rightarrow\left|x_{1}\right|>\left|x_{2}\right|,
$$

contradicting $|a| \leqq|b|$. Therefore, $z \leqq \pi$. Integrating from $T=0$ we get

$$
\left(T\left(x_{1}\right)=T\left(x_{2}\right) \wedge x_{1}<x_{2}\right) \Rightarrow x_{1}-a \geqq b-x_{2} \Rightarrow z \geqq(a+b) / 2 .
$$

We shall prove that (5) implies (4). Assume, first, $b \leqq \pi$. Then $h(x)$ is strictly decreasing in $(a, b)$, and $g(x)$ has exactly one root $y$ in $(a, b)$. Denoting by $G$ a primitive function to $g(4)$ is equivalent to

$$
\int_{x=a}^{x=y} d G T\left(x_{1}(G)\right)<\int_{x=b}^{x=y} d G T\left(x_{2}(G)\right)
$$

where $x_{1}(G)$ and $x_{2}(G)$ are the two inverse functions to $G(x)$. It suffices to prove $T\left(x_{1}(G)\right) \leqq T\left(x_{2}(G)\right)$ for all values of $G$ between $G(a)=G(b)$ and $G(y)$, with strict inequality for at least one value of $G$. In fact, if we can show

$$
x_{1}(G)+x_{2}(G) \leqq a+b
$$

(strict inequality for at least one $G$ ), evidently (5) implies $T\left(x_{1}(G)\right.$ ) $T\left(x_{2}(G)\right)$; either $x_{2}(G) \leqq z$, and the inequality is true, or $x_{2}(G)>z \geqq$ $(a+b) / 2$, and $T\left(x_{1}\right) \leqq T\left(a+b-x_{1}\right) \leqq T\left(x_{2}\right)$, as postulated. 
According to definition, $G\left(x_{1}(G)\right)=G\left(x_{2}(G)\right)$, i.e.

$$
\int_{x_{1}}^{x_{2}} d x g(x)=0 \quad \text { or } \quad \frac{\int_{x_{1}}^{x_{2}} d x h(x)}{x_{2}-x_{1}}=\frac{\int_{a}^{b} d x h(x)}{b-a}
$$

The function

$$
h_{1}\left(x_{1}\right)=\int_{x_{1}}^{x_{2}} d x h(x) /\left(x_{2}-x_{1}\right)
$$

has the derivative

$$
h_{1}^{\prime}\left(x_{1}\right)=\left(h_{1}\left(x_{1}\right)-h\left(x_{1}\right)\right) /\left(x_{2}-x_{1}\right)
$$

as $h_{1}\left(x_{1}\right)$ is the average of $h(x)$ over $\left(x_{1}, x_{2}\right)$, it is strictly decreasing, since $h(x)$ is.

Therefore, if (6) is not satisfied,

$$
h_{1}\left(x_{1}\right) \leqq h_{1}\left(a+b-x_{2}\right)
$$

The function

$$
h_{2}(x)=\frac{1}{2 x} \int_{(a+b) / 2-x}^{(a+b) / 2+x} d t h(t)
$$

has the derivative

$h_{2}^{\prime}(x)=\frac{1}{2 x^{2}}\left(x \cdot\left(h\left(\frac{1}{2}(a+b)+x\right)+h\left(\frac{1}{2}(a+b)-x\right)\right)-\int_{(a+b) / 2-x}^{(a+b) / 2+x} d t h(t)\right)$

the numerator vanishes for $x=0$ and has the derivative

$$
x\left(h^{\prime}\left(\frac{1}{2}(a+b)+x\right)-h^{\prime}\left(\frac{1}{2}(a+b)-x\right)\right),
$$

which is positive, since $h^{\prime \prime}(x)=(2+\cos x) /(1-\cos x)^{2}$ is positive.

Therefore $h_{2}(x)$ is strictly increasing, and we have, according to (8) and (9),

$$
\begin{aligned}
h_{1}\left(x_{1}\right) & \leqq h_{1}\left(a+b-x_{2}\right) \\
& =h_{2}\left(x_{2}-\frac{1}{2}(a+b)\right)<h_{2}\left(\frac{1}{2}(b-a)\right) \text { for } x_{2}<b .
\end{aligned}
$$

This contradicts (7), and (6) must be true.

Next, assume $b>\pi$. The function $h(x)$ is increasing for $x>\pi$, and $g(x)$ may have two roots in $(a, b)$. Assume first that $g(x)$ has only one root in this interval. Then we can use the proof above with the complication that $h_{1}\left(x_{1}\right)$ is not decreasing for $x_{2}>\pi$ and $x_{1}$ close to $\pi$. But $x_{1} \leqq \pi$, and $h_{1}(\pi)$ is a local maximum for $h_{1}\left(x_{1}\right)$. In fact, $h_{1}^{\prime}\left(x_{1}\right)=0 \Rightarrow$ $h_{3}\left(x_{1}\right) \equiv \int_{x_{1}}^{x_{2}} d x\left(h(x)-h\left(x_{1}\right)\right)=0$; but $h_{3}^{\prime}\left(x_{1}\right)=-\left(x_{2}-x_{1}\right) h^{\prime}\left(x_{1}\right) \geqq 0$ for $x_{1} \leqq \pi$. 
Therefore $h_{1}^{\prime}$ has one and only one root, $x_{3}<\pi$. We have $2 \pi-x_{2}<x_{3}<\pi$, and always $a+b-x_{2}<x_{3}$; but if $x_{1}>x_{3}$ we have

$$
h_{1}\left(x_{1}\right) \leqq h_{1}(\pi)=h_{1}\left(2 \pi-x_{2}\right)<h_{1}\left(a+b-x_{2}\right),
$$

which is all we need.

Next assume that $g(x)$ has two roots $y_{1}$ and $y_{2}$. Then at some point $y_{0}$ between $a$ and $b$ we must have $G(a)=G\left(y_{0}\right)$, and the inequality (4) can be written

$$
\begin{aligned}
\left(\int_{x=a}^{x=y_{1}} d G T\left(x_{1}(G)\right)-\right. & \left.\int_{x=y_{0}}^{x=y_{1}} d G T\left(x_{2}(G)\right)\right) \\
& +\left(-\int_{x=y_{2}}^{x=y_{0}} d G T\left(x_{3}(G)\right)+\int_{x=y_{2}}^{x=b} d G T\left(x_{4}(G)\right)\right)<0,
\end{aligned}
$$

where $x_{j}(G)(1 \leqq j \leqq 4)$ are the 4 relevant inverse functions to $G(x)$. Following the method above we easily prove $T\left(x_{1}(G)\right) \leqq T\left(x_{2}(G)\right.$ ) (with strict inequality at least for one $G$ ); the proof will be finished, if we can prove $T\left(x_{3}(G)\right) \geqq T\left(x_{4}(G)\right)$. It suffices to prove $y_{0} \geqq \pi$, since $y_{0} \leqq x_{3}(G) \leqq$ $x_{4}(G)$ and $z \leqq \pi$. But $\int_{a}^{y_{0}} d x g(x)=\int_{a}^{b} d x g(x)=0$, i.e.

$$
\frac{1}{b-y_{0}} \int_{y_{0}}^{b} d x h(x)=\frac{1}{b-a} \int_{a}^{b} d x h(x) \text {. }
$$

If $y_{0}<\pi$ we would have $h_{1}\left(y_{0}\right)<h_{1}(a)$, a contradiction.

Finally we must show that condition (5) is fulfilled. I shall use the notation: $\tau$ is the set of tps of degree $n$ and maximal absolute value 1, with real coefficients and $2 n$ real roots. $T_{m, p}(1 \leqq m \leqq 2 n$ and $0<p \leqq 1)$ is the (apart from the sign) unique tp $\in \tau$ satisfying:

(1) 0 is a root of exact multiplicity $m$.

(2) Denoting by $r$ the least positive root of $T_{m, p}^{\prime}$ we have $0<\left|T_{m, p}(r)\right|=$ $p \leqq 1$.

(3) $\left|T_{m, p}(x)\right|=1$, if $x$ equals one of the remaining $2 n-m$ roots of $T_{m, p}^{\prime}$.

The main tools will be two transformations $k_{1, m, p}$ and $k_{2, m, p}$ of one such tp into another. In fact, $k_{1, m, p} T_{m, 1}=T_{m, p}$ and $k_{2, m, p} T_{m-1,1}=T_{m, p}$.

First $k_{1, m, p}$ will be investigated. In the next paragraphs we shall for notational convenience replace $T_{m, p}$ by $T$. We still let $r$ denote the smallest positive root of $T^{\prime}$, and we have $|T(r)|=p$. Let $t(x)=T^{\prime}(x) \sin \frac{1}{2} x / \sin \frac{1}{2}(x-r)$ and, for $x \neq r, \delta(x)=-\varepsilon \sin \frac{1}{2} x / \sin \frac{1}{2}(x-r)$. Then for $x \neq r$ we have

$$
T(x+\delta(x))+\varepsilon t(x+\delta(x))=T(x)+O\left(\varepsilon^{2}\right) .
$$

The changes in the extremal values (other than $T(r)$ ) of $T$ will be of 
second order in $\varepsilon$, while the changes in their positions in general will be of first order in $\varepsilon$.

The change $\varepsilon t(r)$ in $T(r)$ will be of first order in $\varepsilon$, and $|T(r)|$ will decrease. Replacing $T(x)$ by $T(x)+\varepsilon t(x)$ we can define a new function $t(x)$ and transform again, etc.

It is seen that $k_{1, m, p}$ can be conceived as an infinite product of infinitesimal transformations of the type described above (for $\varepsilon \rightarrow 0$ ), operating on $T_{m, 1}$ and producing $T_{m, p}$ as end result. Similarly, $k_{2, m, p}$ is generated partly by transformations corresponding to

$$
t(x)=T^{\prime}(x) \sin \frac{x}{2}\left(\frac{1}{\sin \frac{1}{2}(x-r)}-\frac{1}{\sin \frac{1}{2}(x+r)}\right)=T^{\prime}(x) \frac{2 \sin x \sin r / 2}{\cos r-\cos x}
$$

( $T_{m-1,1}$ is hereby transformed into a tp $T_{m-1, p, p} \in \tau$ with 0 a root of multiplicity $m-1$ and $\left|T_{m-1, p, p}(r)\right|=p$, where $r$ is the smallest positive root of $T_{m-1, p, p}^{\prime}$; besides, $\left|T_{m-1, p, p}(x)\right|=\left|T_{m-1, p, p}(-x)\right|$ for $\left.0 \leqq x \leqq \pi\right)$, partly by transformations corresponding to $t(x)=-T^{\prime}(x) \sin \frac{1}{2} x / \sin \frac{1}{2}(x+r)$, whereby $T_{m-1, p, p}$ is transformed into $T_{m, p}$.

We shall prove (5) for every root interval $(a, b)$ and every $m \geqq 1$. Let $a \leqq x \leqq b$. Replacing $T$ by $T+\varepsilon t$ will, as noted above, apart from quantities of second order in $\varepsilon$, move a point $(x, T(x))$ to the point $(x+\varepsilon \delta(x), T(x))$, where (for $x \neq r) \delta(x)=-t(x) / T^{\prime}(x)$ (or its limit). $T^{\prime}$ will be divided by $1+\varepsilon \delta^{\prime}(x)$.

Consider, first, the case $t(x)=T^{\prime}(x) \sin \frac{1}{2} x / \sin \frac{1}{2}(x-r)$, corresponding to $k_{1, m, p} ; \delta^{\prime}(x)=\sin \frac{1}{2} r /(1-\cos (x-r))$. Then $\left|x_{1}-r\right|<\left|x_{2}-r\right| \Rightarrow \delta^{\prime}\left(x_{1}\right)>$ $\delta^{\prime}\left(x_{2}\right)$. For $m=1$ we can only use a $k_{1}$-transformation. But this suffices, since $T_{1, p}$ is an even function of $x-r$ : For the root intervals containing $r$ and $r-\pi(5)$ is satisfied with equality. Moreover, the transformation $k_{1,1, p}$ makes the left endpoint of the interval containing $\pi+r$ move from $\pi$ towards the left, and for $p \rightarrow 0, z \rightarrow \pi$. But then for $x_{1}$ and $x_{2}$ belonging to one of the other root intervals we have $\left|x_{1}\right|<\left|x_{2}\right| \Rightarrow\left|x_{1}-r\right|<\left|x_{2}-r\right|$, and (5) is satisfied.

This was the first part of a proof of (5) by induction on $m$.

Let $m>1$. Assume that (5) is satisfied for $T_{k, p}$ with $1 \leqq k \leqq m-1$ and $0<p \leqq 1$ (note that $\lim _{p \rightarrow 0} T_{m-1, p}=T_{m, 1}$, if signs are chosen suitably). Assume that there is a smallest value $p_{0}>0$ for $p$, for which (5) is valid for $T_{m, p}$. Now we need some information concerning the transformations generating $k_{2, m, p}$. Corresponding to $t(x)=-T^{\prime}(x) \sin \frac{1}{2} x / \sin \frac{1}{2}(x+r)$ we find $\delta^{\prime}(x)=\sin \frac{1}{2} r /(1-\cos (x+r))$ so that $\left|x_{1}+r\right|<\left|x_{2}+r\right| \Rightarrow \delta^{\prime}\left(x_{1}\right)>\delta^{\prime}\left(x_{2}\right)$. Corresponding to

$$
t(x)=T^{\prime}(x) \sin \frac{x}{2}\left(\frac{1}{\sin \frac{1}{2}(x-r)}-\frac{1}{\sin \frac{1}{2}(x+r)}\right)
$$


we find

$$
\delta^{\prime}(x)=\sin \frac{r}{2}\left(\frac{1}{1-\cos (x-r)}+\frac{1}{1-\cos (x+r)}\right),
$$

which is an even function of $x$ and a decreasing function of $|x|$ for $|x|>r$.

There are now two subcases.

(1) $m$ even. $T_{m, 1}^{\prime}(\pi)=0$, and $k_{1, m, p}$ moves all points to the left, so that $\lim _{p \rightarrow 0} T_{m, p}(\pi)=0$. In particular, for all root intervals $(a, b)$ with $0<z \leqq \pi$, (5) is fulfilled for $p<p_{0}$ too. For intervals with $-\pi<z<0, k_{2, m, p}$ is seen to work, so that (5) is fulfilled here too, and we obtain a contradiction.

(2) $m$ odd. Here $k_{1, m, p}$ works as before, if $0<z<\pi$, and $k_{2, m, p}$ takes care of the remaining intervals. The interval containing $-\pi$ may need a more elaborated argument. In fact, after the first part of $k_{2, m, p}$, $T_{m-1, p, p}^{\prime}(-\pi)=0$; during the last part of $k_{2, m, p}$ all points are moved to the right, so that for $x_{1}, x_{2}$ belonging to the interval in question $\left|x_{1}\right|<\left|x_{2}\right| \Rightarrow\left|x_{1}+r\right|<\left|x_{2}+r\right| \Rightarrow \delta^{\prime}\left(x_{1}\right)>\delta^{\prime}\left(x_{2}\right)$, so that (5) is fulfilled also for $p<p_{0}$.

(B3) We now know that $T$ has only simple roots. Assume that $T$ has a regular interval. $T^{\prime}$ has a root in this interval, which we set equal to zero. Putting $t(x)=-T^{\prime}(x) \cot (x / 2)$ we get the conditions (4) and (5) as before. If $T$ has only one regular interval, we use the transformation generated by $t(x)=T^{\prime}(x) \cot (x / 2)$ to prove that (5) is satisfied. Next we consider the case where $T$ has exactly two regular intervals, and we assume first that these intervals have one endpoint in common. One of the two corresponding roots for $T^{\prime}$ is zero, and we can assume that the other one $(r$, say) is positive. Then we first use the transformation described above to produce the right value for $x=0$, next we use the transformation generated by $t(x)=T^{\prime}(x) \sin \frac{1}{2} x / \sin \frac{1}{2}(x-r)$ to show that (5) is fulfilled for $0<z<\pi$. During the first step the distance between $\pi$ and the next extremum to its right becomes greater than the smallest positive root of $T^{\prime}$. During the last step we find for the movement of the smallest positive root of $T^{\prime}$,

$$
\delta(r)=-\left(\cos \frac{r}{2}+\frac{T^{\prime \prime \prime}(r)}{T^{\prime \prime}(r)} \sin \frac{r}{2}\right) ;
$$

but (5) implies $T^{\prime \prime \prime}(r) / T^{\prime \prime}(r)>0$, so that $|\delta(r)|>|\delta(r-\pi)|$, where the last symbol has the original meaning. It follows that the root-interval immediately to the right of the interval containing $-\pi$ has a root for $T^{\prime}$ whose distance from $-\pi$ is greater than $r$. But an interchange of the rôle of the two regular intervals excludes the possibility $r-\pi<z<0$.

There remains the case where $T$ has two regular intervals $\left(x_{1}, x_{2}\right)$ and $\left(x_{3}, x_{4}\right)$ with no endpoint in common. Here the root set of $t$ is specified as in (B1): If none of the $x_{j}$ coincides with $a$ or $b$, the latter and $z$ shall 
be double roots and the $x_{j}$ nonroots. If there is coincidence the corresponding root is kept simple.

Conclusion. A tp $T \in \tau$ maximizing $f$ has only simple roots, and $|T(x)|$ assumes its maximal value in all roots for $T^{\prime}$, i.e. $T(x)$ is proportional to a sine function, as postulated.

\section{REFERENCE}

1. P. Erdös, Note on some elementary properties of polynomials, Bull. Amer. Math. Soc. 46 (1940), 954-958 (see p. 957). MR 2, 242.

Fynsvej 52, DK 4000 Roskilde, Denmark 